\title{
Rescue Living-donor Liver Transplantation for Liver Failure Following Hepatectomy for Hepatocellular Carcinoma
}

\author{
See Ching Chan ${ }^{a, b}$ William Wei Sharr ${ }^{a}$ Albert Chi Yan Chan ${ }^{a}$ \\ Kenneth Siu Ho Chok ${ }^{a}$ Chung Mau Loa,b \\ aDepartment of Surgery, and 'bState Key Laboratory for Liver Research, The University of \\ Hong Kong, Hong Kong, SAR, China
}

\section{Key Words}

Hepatocellular carcinoma $\cdot$ Liver failure $\cdot$ Living-donor liver transplantation · Rescue

\begin{abstract}
Liver failure following major hepatectomy for hepatocellular carcinoma is a known but uncommon mode of early treatment failure. When post-hepatectomy liver failure becomes progressive, the only effective treatment for rescuing the patient is liver transplantation. Deceased-donor liver transplantation in this situation is often not feasible because of the shortage of deceased-donor liver grafts. Proceeding with living-donor liver transplantation is an ethical challenge because of the possibility of donor coercion. In addition, tumor status, as confirmed by histopathological examination of the resected specimen, may indicate aggressive cancer that warns against rescue transplantation because of the increased chance of tumor recurrence. Here we describe four cases of rescue living-donor liver transplantation for liver failure after hepatectomy for hepatocellular carcinoma. The patients all survived the transplantation and were free from tumor recurrence after follow-up periods ranging from 6 months to 9 years. Our experience has shown that rescue living-donor liver transplantation for post-hepatectomy liver failure is feasible. Tumor status should be considered carefully because large tumors and tumors with macrovascular invasion are strong contraindications to rescue living-donor liver transplantation.

Copyright @ 2013 S. Karger AG, Basel
\end{abstract}

See Ching Chan, MBBS, MS, PhD, MD Department of Surgery, The University of Hong Kong 102 Pok Fu Lam Road, Hong Kong, SAR (China)

Tel. +852 2255 3025, E-Mail seechingchan@gmail.com 


\section{Introduction}

Liver resection (LR) is the most common form of curative treatment for hepatocellular carcinoma (HCC) [1]. Patients with resectable HCC who undergo major hepatectomy have a hospital mortality rate of less than $5 \%$ and a 5-year survival rate of $50 \%$ [2]. However, for patients with liver cirrhosis and compromised liver function, liver failure after major hepatectomy remains a significant issue and is a major cause of hospital mortality $[3,4]$. Accurate remnant liver volumetric analysis [5], liver function assessment [6], preoperative ipsilateral portal vein embolization [7], and parenchyma-sparing hepatectomy may lower the incidence of post-hepatectomy liver failure (PHLF) [8].

When PHLF becomes progressive, the only available life-saving measure is liver transplantation (LT). When a deceased-donor liver graft is not available or cannot be allocated to a patient with PHLF because of unsuitable tumor status, living-donor liver transplantation (LDLT) [9] can be considered if there is no strong contraindication such as major vascular invasion by HCC. Here we describe four cases of rescue LDLT for liver failure following hepatectomy for HCC. The biological and ethical issues of such last-resort LDLTs are also discussed.

\section{Patients and Methods}

Over 800 LRs, $57 \%$ of which were major hepatectomies, have been performed at our hospital, a tertiary center for referral for management of HCC [10]. Data on consecutive patients who underwent LR or LT at our hospital were prospectively entered into two computerized databases. Patients who developed irreversible liver failure and those who underwent LT were identified. Resected HCC specimens were examined by histopathologists. Data regarding tumor size and number, satellite tumors, tumor cell differentiation, and vascular invasion (macro or micro) were recorded. Patients who had progressive PHLF but no strong contraindication were offered LT. Because of the scarcity of deceased-donor liver grafts in our region, LDLT was proposed in such urgent situations.

Donor work-up was performed as per our protocol. High-urgency rescue LDLT was enabled by an expedient preparatory process [11]. Graft size estimation was done by volumetric analysis of the potential donor's right liver on computed tomography [12]. The potential donors, recipients, and their families were informed that the mortality rate is about $0.5 \%$ for donors of the right liver including the middle hepatic vein, and the long-term survival rate of recipients is 50-70\% [13]. LDLT was not offered to patients with an estimated survival rate of less than 50\%. Histopathology reports of the resected tumor specimens provided evidence of the absence or presence of vascular invasion by HCC. Computed tomography of the recipient's brain was performed shortly before commencement of LDLT to confirm the absence of cerebral edema, a condition that would result in transplantation failure.

The donor right hepatectomy [14] and recipient operation [15] are described in detail elsewhere. Immunosuppression included interleukin-2 receptor antagonist and was steroid free [16]. Hepatitis B prophylaxis did not contain hepatitis B immunoglobulin and was administered in the form of a monoagent oral nucleoside analogue [17]. After transplantation, computed tomography was done every 3-4 months and serum alpha-fetoprotein levels were checked to monitor HCC status.

\section{Results}

As shown in table 1, the four cases of progressive PHLF occurred in $2003(\mathrm{n}=1)$ and $2011(\mathrm{n}=3)$. All four patients were carriers of the hepatitis B virus. Patient 2 had moderate cirrhosis and the other patients had mild cirrhosis as judged at LR. Patient 3 received LR at another hospital, and did not undergo a preoperative indocyanine green test. All patients underwent right hepatectomy without Pringle maneuver, except patient 4, whose liver tran- 

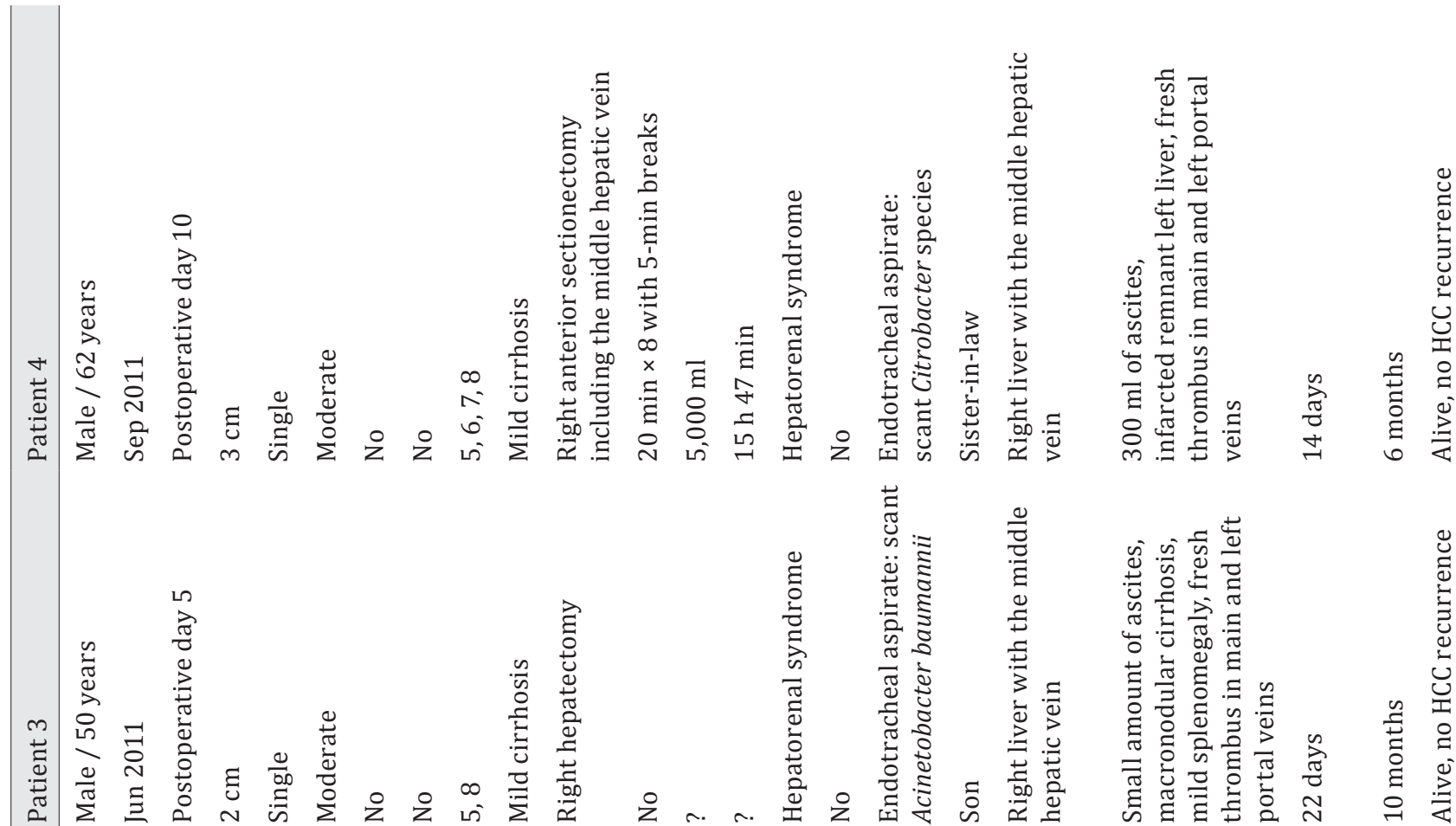

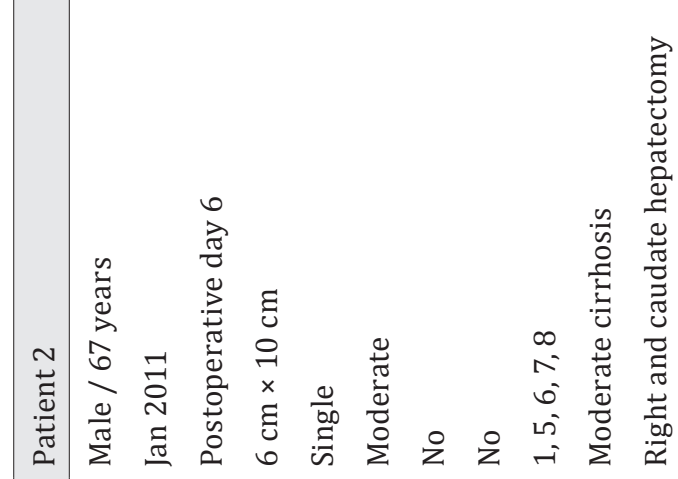
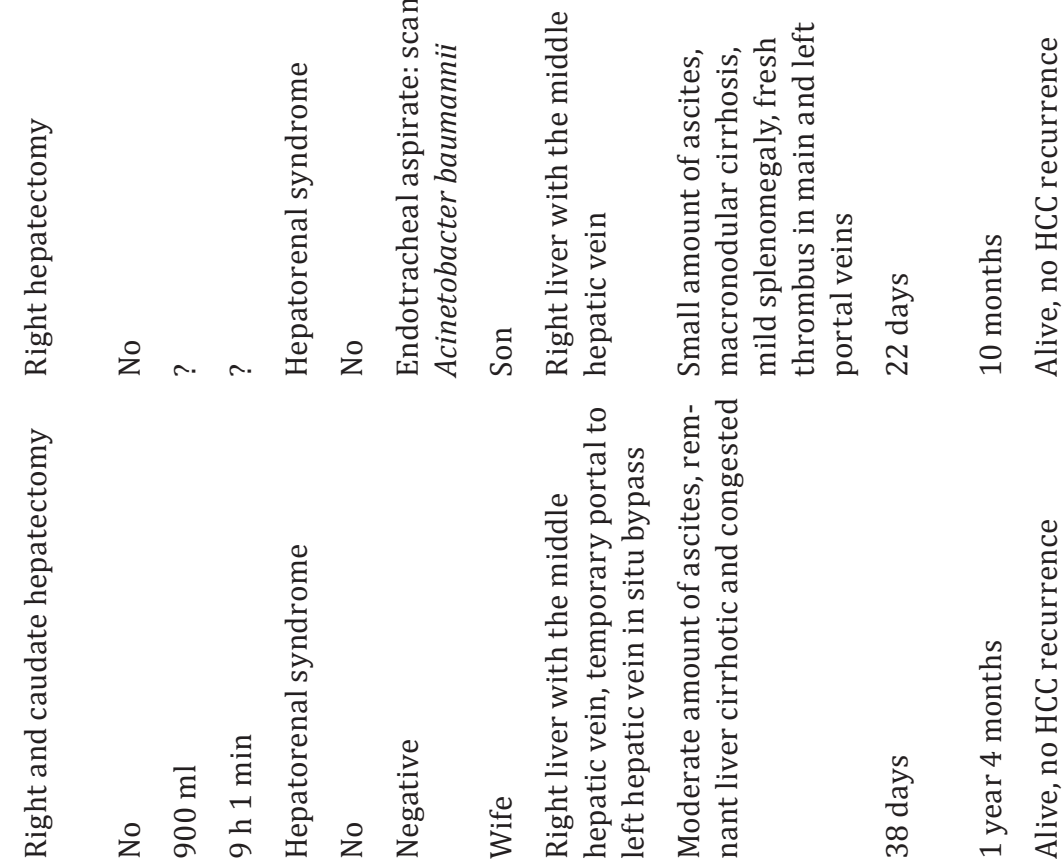
section was difficult. Patients 1 and 4 had significant blood loss of 4,500 ml and 5,000 ml, respectively.

The development of progressive PHLF was unexpected in each patient but became apparent in the early postoperative period. The development of PHLF was rapid, and all patients presented with hepatorenal syndrome. LDLT was performed on postoperative days $7,6,5$, and 10 respectively. The decision to proceed with LDLT for patient 4 was made on postoperative day 6, but there was no suitable donor from among his first-degree relatives. His sister-in-law came forward as a donor on postoperative day 9 , and the donor operation was performed $12 \mathrm{~h}$ after the completion of donor work-up.

All donors donated their right liver with the middle hepatic vein. The donor operations were standard. The recipient operations were not difficult because the right liver had been removed during LR. When implanting the graft in patient 2, hypertensive myopathy was modified with the construction of a temporary portal systemic shunt using a ringed Gortex graft. This connected the native main portal vein to the common trunk of the middle and left hepatic veins to improve hemodynamic stability during the anastomosis of the hepatic vein and the inferior vena cava. The patency of the latter was restored before taking down the shunt. Extracorporeal venovenous bypass was not performed, based on our experience [18]. All four patients survived and did not have recurrence of HCC after follow-up periods ranging from 6 months to 9 years. All the four living donors returned to their normal life and activities.

\section{Discussion}

It was fortunate that the four patients did not have any strong contraindications to LT such as uncontrolled sepsis or major vascular invasion by HCC. Although PHLF is irreversible and progressive, the patients' physical conditions were not prohibitive of LT. The four cases of emergency LDLT were successful in reviving the patients. Histopathological examination of the resected specimens demonstrated no features indicative of a high chance of HCC recurrence after transplantation. In fact, HCC recurrence in such situations is uncommon.

HCC patients within the standard criteria or the "up-to-7" criteria who undergo LT despite the presence of microvascular invasion have a 5-year survival rate of 85\% [19], which is comparable to the survival rate for LT recipients with no HCC. However, the survival outcome of salvage LT for HCC recurrence is poor [20]. Nonetheless, LT has been proposed [21] and is practiced for patients who have tumors with a high chance of recurrence after resection. Even patients who have three to five or even more factors associated with tumor recurrence are advised to undergo LT. Recurrence-associated factors include microscopic vascular invasion, satellite nodules, tumor size $>3 \mathrm{~cm}$, poorly differentiated tumor, and liver cirrhosis [22].

Based on the normal course of biochemical liver function tests after LR, a simple and easily applicable definition of PHLF was developed by the International Study Group of Liver Surgery. Furthermore, a grading of severity of PHLF was proposed based on the disease's impact on the clinical management of patients [23]. Grade-A PHLF requires no change of clinical management. The clinical management of patients with grade-B PHLF deviates from the regular course but does not require invasive therapy. The need for invasive treatment defines grade-C PHLF. Immediate LT should be considered seriously for grade-C PHLF. It has been reported that a preoperative Model for End-stage Liver Disease score of 11 or higher, major hepatectomy, and a rise in the score on postoperative days 3-5 are significant factors for PHLF, but it should be noted that only $4.5 \%$ of the patients in our series underwent major hepatectomy [24]. 
Rescue LT had been practiced elsewhere [24, 25]. In a series at the University of California, Los Angeles, 11 patients were considered and seven were listed for LT. The four excluded patients had advanced malignancy, uncontrolled sepsis, ischemic heart disease, or multipleorgan failure. The seven listed patients underwent deceased-donor LT [25].

The potential for LDLT donors to feel some level of coercion in such a high-urgency situation is of concern [26]. The rapidity required for decision making is the main argument against such donation. In LDLT, the double equipoise model is applicable to balancing recipient benefit against donor risk [27]. A patient with PHLF needs a LT to survive, and if there is a chronic shortage of deceased-donor liver grafts, the chance of survival is virtually zero. Thus, the recipient benefit of LDLT is tremendous [28]. There is also evidence that the donor's quality of life is worse if the recipient suffers complications [29]. Young donors and donors who have greater pre-donation concern over complications are more likely to have poorer outcomes after donation [30,31]. Since the indications for LR are not restricted by tumor size or number, there is a question as to whether these patients should be transplanted after the development of irreversible PHLF. Given the rigid criteria (e.g., Milan or UCSF criteria) for the allocation of liver grafts, deceased-donor LT for patients beyond the standard criteria is not feasible. HCC that is slightly beyond these criteria in terms of size or number, however, does not preclude LDLT as long as the chance of long-term survival is not too low. The enthusiasm of the donor and recipient is then of great importance [32].

Rescue LDLT for PHLF may open up the treatment logistics of a planned salvage LT when the patient course indicates such a possibility. A more aggressive approach to major hepatectomy can be executed in patients with small resectable HCC and compromised liver function. This can be carried out on the premise that the potential donor is already worked up and is unlikely to withdraw from donation. LDLT can also be preemptive if PHLF is determined early and accurately. The combination of a prothrombin level of $<50 \%$ and a serum total bilirubin level of $>50 \mu \mathrm{mol} / \mathrm{L}$ on postoperative day 5 is a strong predictive factor for mortality. In patients with significant morbidity, this " $50-50$ " criterion is met $3-8$ days before clinical evidence of complications. It is a simple, early, and accurate predictor of more than half of deaths after hepatectomy. This criterion can be identified early enough, before clinical evidence of complications, for specific intervention to be applied in due time [33]. Patient 3 underwent LDLT within a week of LR, while Patient 4 underwent LDLT after 10 days because no suitable donor was available earlier. Donor work-up was also expedient. Trace amounts of Acinetobacter were cultured from the endotracheal aspirates of two patients.

At many centers, LDLT has become the standard treatment for acute liver failure. In the setting of PHLF, the general principle of this form of treatment is applied. Tumor factors should be considered carefully because large tumors and tumors with macrovascular invasion as determined in LR specimens are strong contraindications to rescue LDLT. However, our experience has shown that emergency rescue LDLT for PHLF is feasible.

\section{References:}

1 Belghiti J, Fuks D: Liver resection and transplantation in hepatocellular carcinoma. Liver Cancer 2012;1:71-82.

$\checkmark 2$ Chan SC, Liu CL, Lo CM, Lam CM, Poon RT, Yuen WK, et al: Value of live donor liver transplantation experience in major hepatectomy for hepatocellular carcinoma. Arch Surg 2003;138:265-271.

-3 Jarnagin WR, Gonen M, Fong Y, DeMatteo RP, Ben-Porat L, Little S, et al: Improvement in perioperative outcome after hepatic resection: analysis of 1,803 consecutive cases over the past decade. Ann Surg 2002;236:397-406; discussion -7 .

-4 Poon RT, Fan ST, Lo CM, Liu CL, Lam CM, Yuen WK, et al: Improving perioperative outcome expands the role of hepatectomy in management of benign and malignant hepatobiliary diseases: analysis of 1222 consecutive patients from a prospective database. Ann Surg 2004;240:698-708; discussion -10. 
$\checkmark 5$ Chan SC, Liu CL, Lo CM, Lam BK, Lee EW, Wong Y, et al: Estimating liver weight of adults by body weight and gender. World J Gastroenterol 2006;12:2217-2222.

6 Fan ST: Liver functional reserve estimation: state of the art and relevance for local treatments: the Eastern perspective. J Hepatobiliary Pancreat Sci 2010;17:380-384.

7 Abdalla EK: Portal vein embolization (prior to major hepatectomy) effects on regeneration, resectability, and outcome. J Surg Oncol 2010;102:960-967.

-8 Reddy SK, Barbas AS, Turley RS, Steel JL, Tsung A, Marsh JW, et al: A standard definition of major hepatectomy: resection of four or more liver segments. HPB (Oxford)2011;13:494-502.

$\$ 9$ Cheah YL, Chow P: Liver transplantation for hepatocellular carcinoma: an appraisal of current controversies. Liver Cancer 2012;1:183-189.

10 Fan ST, Mau Lo C, Poon RT, Yeung C, Leung Liu C, Yuen WK, et al: Continuous improvement of survival outcomes of resection of hepatocellular carcinoma: a 20-year experience. Ann Surg 2011;253:745-758.

$\checkmark 11$ Chan SC, Fan ST, Liu CL, Lo CM, Lam BK, Lee EW: Working up donors for high-urgency and elective adultto-adult live donor liver transplantation. Liver Transpl 2007;13:509-515.

12 Chan SC, Lo CM, Chok KS, Sharr WW, Cheung TT, Tsang SH, et al: Validation of graft and standard liver size predictions in right liver living donor liver transplantation. Hepatol Int 2011;5:913-917.

13 Chan SC, Fan ST, Lo CM, Liu CL, Wong J: Toward current standards of donor right hepatectomy for adultto-adult live donor liver transplantation through the experience of 200 cases. Ann Surg 2007;245:110117.

14 Chan SC, Lo CM, Liu CL, Wong Y, Fan ST, Wong J: Tailoring donor hepatectomy per segment 4 venous drainage in right lobe live donor liver transplantation. Liver Transpl 2004;10:755-762.

15 Chan SC, Lo CM, Fan ST: Simplifying living donor liver transplantation. Hepatobiliary Pancreat Dis Int 2010;9:9-14.

16 Liu CL, Fan ST, Lo CM, Chan SC, Ng IO, Lai CL, et al: Interleukin-2 receptor antibody (basiliximab) for immunosuppressive induction therapy after liver transplantation: a protocol with early elimination of steroids and reduction of tacrolimus dosage. Liver Transpl 2004;10:728-733.

17 Fung J, Cheung C, Chan SC, Yuen MF, Chok KS, Sharr W, et al: Entecavir monotherapy is effective in suppressing hepatitis B virus after liver transplantation. Gastroenterology 2011;141:1212-1219.

18 Fan ST, Yong BH, Lo CM, Liu CL, Wong J: Right lobe living donor liver transplantation with or without venovenous bypass. Br J Surg 2003;90:48-56.

19 Chan SC, Fan ST, Chok KS, Cheung TT, Chan AC, Fung JY, et al: Survival advantage of primary liver transplantation for hepatocellular carcinoma within the up-to-7 criteria with microvascular invasion. Hepatol Int 2012;6:646-656.

20 Adam R, Azoulay D, Castaing D, Eshkenazy R, Pascal G, Hashizume K, et al: Liver resection as a bridge to transplantation for hepatocellular carcinoma on cirrhosis: a reasonable strategy? Ann Surg 2003;238:508-518, discussion 18-19.

-21 Sala M, Fuster J, Llovet JM, Navasa M, Sole M, Varela M, et al: High pathological risk of recurrence after surgical resection for hepatocellular carcinoma: an indication for salvage liver transplantation. Liver Transpl 2004;10:1294-1300.

-22 Fuks D, Dokmak S, Paradis V, Diouf M, Durand F, Belghiti J: Benefit of initial resection of HCC followed by transplantation in case of recurrence: An intention-to-treat analysis. Hepatology 2012;55:132-140.

-23 Rahbari NN, Garden OJ, Padbury R, Brooke-Smith M, Crawford M, Adam R, et al: Posthepatectomy liver failure: a definition and grading by the International Study Group of Liver Surgery (ISGLS). Surgery 2011;149:713-724.

24 Cucchetti A, Ercolani G, Cescon M, Ravaioli M, Zanello M, Del Gaudio M, et al: Recovery from liver failure after hepatectomy for hepatocellular carcinoma in cirrhosis: meaning of the model for end-stage liver disease. J Am Coll Surg 2006;203:670-676.

25 Otsuka Y, Duffy JP, Saab S, Farmer DG, Ghobrial RM, Hiatt JR, et al: Postresection hepatic failure: successful treatment with liver transplantation. Liver Transpl 2007;13:672-679.

-26 Campsen J, Blei AT, Emond JC, Everhart JE, Freise CE, Lok AS, et al: Outcomes of living donor liver transplantation for acute liver failure: the adult-to-adult living donor liver transplantation cohort study. Liver Transpl 2008;14:1273-1280.

27 Cronin DC 2nd, Millis JM, Siegler M: Transplantation of liver grafts from living donors into adults-too much, too soon. N Engl J Med 2001;344:1633-1637.

28 Durand F, Belghiti J, Troisi R, Boillot O, Gadano A, Francoz C, et al: Living donor liver transplantation in high-risk vs. low-risk patients: optimization using statistical models. Liver Transpl 2006;12:231-239.

-29 Kim-Schluger L, Florman SS, Schiano T, O’Rourke M, Gagliardi R, Drooker M, et al: Quality of life after lobectomy for adult liver transplantation. Transplantation 2002;73:1593-1597.

-30 DuBay DA, Holtzman S, Adcock L, Abbey S, Greenwood S, Macleod C, et al: Adult right-lobe living liver donors: quality of life, attitudes and predictors of donor outcomes. Am J Transplant 2009;9:1169-1178.

-31 Holtzman S, Adcock L, Dubay DA, Therapondos G, Kashfi A, Greenwood S, et al: Financial, vocational, and interpersonal impact of living liver donation. Liver Transpl 2009;15:1435-1442.

-32 Grant D, Fisher RA, Abecassis M, McCaughan G, Wright L, Fan ST: Should the liver transplant criteria for hepatocellular carcinoma be different for deceased donation and living donation? Liver Transpl 2011;17(Suppl 2):S133-S138.

33 Balzan S, Belghiti J, Farges 0, Ogata S, Sauvanet A, Delefosse D, et al: The “50-50 criteria” on postoperative day 5: an accurate predictor of liver failure and death after hepatectomy. Ann Surg 2005;242:824-828, discussion 8-9. 